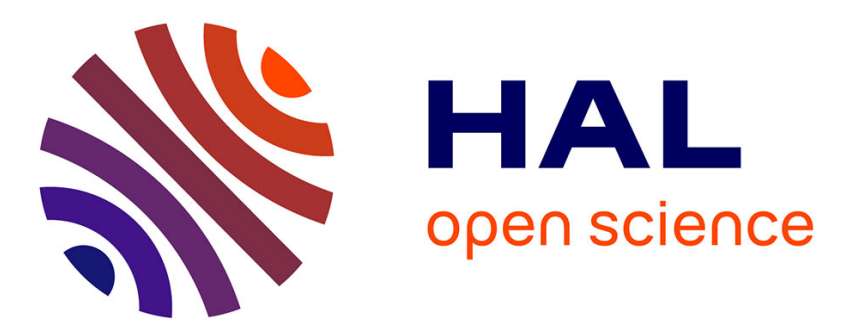

\title{
Forecast and Tracking the Evolution of Cloud Clusters (ForTraCC) Using Satellite Infrared Imagery: Methodology and Validation
}

Daniel Alejandro Vila, Luiz Augusto Toledo Machado, Henri Laurent, Ines Velasco

\section{To cite this version:}

Daniel Alejandro Vila, Luiz Augusto Toledo Machado, Henri Laurent, Ines Velasco. Forecast and Tracking the Evolution of Cloud Clusters (ForTraCC) Using Satellite Infrared Imagery: Methodology and Validation. Weather and Forecasting, 2008, 23 (2), pp.233 à 245. 10.1175/2007WAF2006121.1 . insu-00389162

\section{HAL Id: insu-00389162 \\ https://hal-insu.archives-ouvertes.fr/insu-00389162}

Submitted on 11 Jun 2021

HAL is a multi-disciplinary open access archive for the deposit and dissemination of scientific research documents, whether they are published or not. The documents may come from teaching and research institutions in France or abroad, or from public or private research centers.
L'archive ouverte pluridisciplinaire HAL, est destinée au dépôt et à la diffusion de documents scientifiques de niveau recherche, publiés ou non, émanant des établissements d'enseignement et de recherche français ou étrangers, des laboratoires publics ou privés. 


\title{
Forecast and Tracking the Evolution of Cloud Clusters (ForTraCC) Using Satellite Infrared Imagery: Methodology and Validation
}

\author{
Daniel Alejandro Vila \\ Cooperative Institute of Climate Studies, University of Maryland, College Park, College Park, Maryland \\ Luiz Augusto Toledo Machado \\ Centro de Previsão de Tempo e Estudos Climáticos, Instituto Nacional de Pequisas Espaciais, Cachoeira Paulista, São Paulo, Brazil \\ HENRI LAURENT \\ Institut de Recherche pour le Developpement, Paris, France \\ INÉS VELASCO \\ Departamento de Ciencias de la Atmósfera, y los Océanos, Facultad de Ciencias Exactas y Naturales, Universidad de Buenos Aires, \\ Buenos Aires, Argentina
}

(Manuscript received 27 December 2006, in final form 23 May 2007)

\begin{abstract}
The purpose of this study is to develop and validate an algorithm for tracking and forecasting radiative and morphological characteristics of mesoscale convective systems (MCSs) through their entire life cycles using geostationary satellite thermal channel information $(10.8 \mu \mathrm{m})$. The main features of this system are the following: 1) a cloud cluster detection method based on a threshold temperature $(235 \mathrm{~K}), 2)$ a tracking technique based on MCS overlapping areas in successive images, and 3) a forecast module based on the evolution of each particular MCS in previous steps. This feature is based on the MCS's possible displacement (considering the center of the mass position of the cloud cluster in previous time steps) and its size evolution. Statistical information about MCS evolution during the Wet Season Atmospheric Mesoscale Campaign (WETAMC) of the Large-Scale Biosphere-Atmosphere Experiment in Amazonia (LBA) was used to obtain area expansion mean rates for different MCSs according to their lifetime durations. This nowcasting tool was applied to evaluate the MCS displacement and size evolution over the Del Plata basin in South America up to 120 min with 30-min intervals. The Forecast and Tracking the Evolution of Cloud Clusters (ForTraCC) technique's performance was evaluated based on the difference between the forecasted and observed images. This evaluation shows good agreement between the observed and forecast size and minimum temperature for shorter forecast lead times, but tends to underestimate MCS size (and overestimate the minimum temperature) for larger forecast lead times.
\end{abstract}

\section{Introduction}

Mesoscale convective systems (MCSs) are responsible for most of the warm-season rainfall over tropical South America (Velasco and Fritsch 1987; Vila 2005), the Sahelian region (Mathon and Laurent 2001), and various regions of the world. They are also responsible for some extreme weather conditions, and the fre-

Corresponding author address: Daniel Alejandro Vila, 2207 Computer and Space Bldg., University of Maryland, College Park, College Park, MD 20742.

E-mail: dvila@essic.umd.edu quency of convective systems is the principal factor in determining interannual climate variability over western Africa (Le Barbé et al. 2002).

Nevertheless, in spite of their substantial contribution to the production of significant weather, these systems are not forecasted very well (Corfidi et al. 1996). Knowledge of convective systems' cloud processes and evolution is important for understanding weather and climate, particularly over the tropics. This issue is an essential criterion for improving forecasting and, following this thought, reducing our vulnerability to extreme weather damage.

Infrared imagery from meteorological satellites has 
been broadly used to study the behavior of the cloud systems associated with deep convection for many years (e.g., Houze 1977; Maddox 1980). The main advantage of this approach is that for most of the globe the best statistics can only be obtained from satellite observations. Such a satellite survey would provide the statistics of MCSs covering the range of meteorological conditions needed to generalize the results and, on the other hand, only satellite observations can actually cover the very large range of space and time scales, including MCSs larger than a few hundred kilometers in size, and their interactions with each other and with the planetary-scale atmospheric circulation (Machado et al. 1998).

The identification of predictor parameters of MCS evolution, based on its previous life stage, could make a significant contribution to a nowcasting scheme and can provide important information for mesoscale model initialization (Machado and Laurent 2004).

Since this short-term forecast methodology is based on MCS evolution, it is important to address how cloud tracking techniques works in order to understand the link between these two processes (track and forecast).

From the pioneer works of Woodley et al. (1980) to Machado and Laurent (2004), many authors all around the world have been working with different automated cloud cluster tracking methods. Two methodologies have been extensively used to track clouds using satellite imagery: image overlapping and spatial correlation patterns. Williams and Houze (1987) made an extensive study over the western Pacific Ocean during the Winter Monsoon Experiment and introduced forward and backward tracking, while Mathon and Laurent (2001) used infrared satellite imagery to track MCSs in the Sahelian region during the rainy season. Morel and Senesi (2002) developed a tracking technique [rapid development thunderstorm (RDT)] to track MCSs over Europe using an adaptive temperature threshold of infrared images. Over the American continent, the most recent study of MCS characteristics using a similar tracking technique was carried out by Machado et al. (1998). Carvalho and Jones (2001) used a spatial correlation approach to track MCSs in the Amazonian region, while Scofield et al. (2004) performed a similar technique to determinate the clouds motion. A very important issue in tracking methodologies (regardless of the tracking scheme used) is that a brightness temperature and a minimum size threshold are required to identify MCSs from infrared thermal channels.

The purpose of this study is to present and validate an algorithm to forecast (up to $120 \mathrm{~min}$ ) the radiative and morphological characteristics of MCSs using ther- mal infrared channel information $(10.8 \mu \mathrm{m})$ from geostationary satellites.

The algorithm is described in section 2, while section 3 aims to validate the results of the methodology. The main findings are summarized in section 4 .

\section{The ForTraCC algorithm}

The Forecasting and Tracking the Evolution of Cloud Clusters (ForTraCC) technique is an algorithm that allows for the tracking of MCS radiative and morphological properties and forecasts the evolution of these physical properties (based on cloud-top brightness temperature) up to $120 \mathrm{~min}$, using infrared satellite imagery $(10.8 \mu \mathrm{m})$.

The main steps of this algorithm are the following: 1) a cloud cluster detection method based on a size and temperature threshold, 2) a statistical module to identify morphological and radiative parameters of each MCS, 3) a tracking technique based on MCS overlapping areas between successive images, and 4) a forecast module based on MCS evolution in previous time steps.

\section{a. MCS identification}

Based on the concept that deep convection penetrates into the upper troposphere, the first step in the analysis is to identify all clouds that have tops well above $9-10 \mathrm{~km}$. Although the selection of a specific temperature threshold to define clouds associated with convective activity is arbitrary, different studies support the idea that brightness temperatures below $245 \mathrm{~K}$ identify satisfactorily convective systems. Maddox (1980) identified mesoscale convective complexes (MCCs) using $T_{\text {ir }}<241 \mathrm{~K}$, while Velasco and Fritsch (1987) use a lower $T_{\text {ir }}$ value (231 or $233 \mathrm{~K}$ ). On the other hand, Machado et al. (1998) proposed a $245-\mathrm{K}$ brightness temperature threshold for MCS identification, while temperatures between 238 and $235 \mathrm{~K}$ have been found during the summer season for northern Argentina (Velasco and Necco 1980) to identify convection. Although the threshold selection will affect the area covered by MCSs (and the total life cycle for a given MCS), there is a near-linear dependence between the cloud size and a given temperature threshold (typically below $245 \mathrm{~K}$ ) in the range of 10-20 K (Machado et al. 1993; Mapes and Houze 1992). In other words, the selection of a brightness temperature threshold in the previously defined range is describing, in mean terms, analogous convective systems. In this study, a brightness temperature threshold of $235 \mathrm{~K}$ has been chosen for MCS detection because this threshold seems to be suitable for detecting clouds associated with convection in different regions of South America, as suggested by 


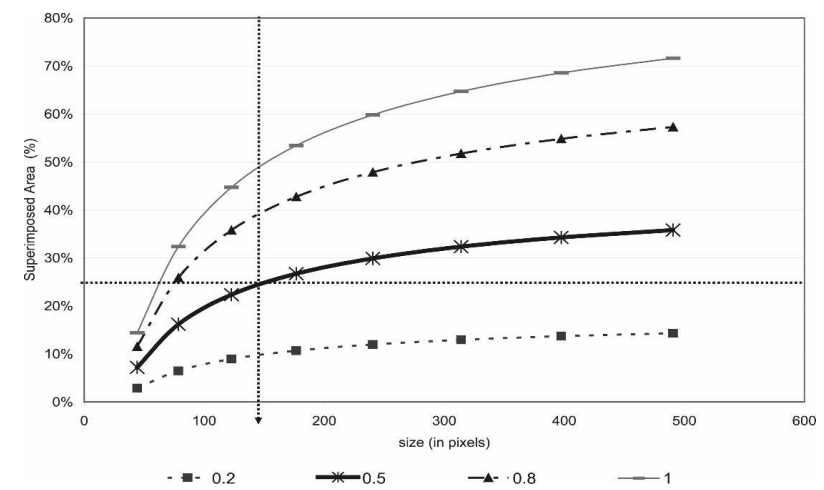

FIG. 1. Percentage of the superimposed area between two ellipses with different eccentricities as a function of the ellipse size. Eccentricities equal to 1 represent a perfect circle.

Laurent et al. (2002), Machado and Laurent (2004), and Carvalho and Jones (2001).

Another important issue is to define the minimum size for detecting a given MCS. Different MCS size thresholds for detecting and tracking MCSs have been proposed by different authors. While Maddox (1980) proposed that a $T_{\text {ir }}<221 \mathrm{~K}$ area should overcome the $50000-\mathrm{km}^{2}$ criterion for MCCs, Torres (2003) proposed a threshold of $25000 \mathrm{~km}^{2}$ for $T_{\text {ir }}<218 \mathrm{~K}$ for a $30-\mathrm{km}$ resolution images study. This selection can affect strongly some of the statistical parameters, such as MCS mean lifetime or MCS genesis location. In this case, a scale analysis was performed to find out the minimum size threshold for detecting and tracking convective systems from successive images for a typical time interval of Geostationary Operational Environmental Satellite (GOES) images (30 min). An ellipseshaped MCS was considered in two successive images. Machado et al. (1998) found that $45 \mathrm{~km} \mathrm{~h}^{-1}$ is a value close to the upper limit of the displacement speed of the center of mass for an MCS with no splits or mergers. So, the distance between the same MCS in two successive images should be less than $22.5 \mathrm{~km}$. Based on this value, the percentage of the superimposed area can be calculated for different MCS sizes and eccentricities (represented by ellipses, in this hypothetical case). Growth or decay processes are not considered. Figure 1 illustrates this situation. Each curve represents different eccentricity types, where 1 is a perfect circle. For a given pair of consecutive MCSs (whose size is known) and for a given eccentricity and for a parallel orientation (worst case), the superimposed area can be obtained from the ordinate axis. Considering a conservative assumption of a minimum overlapping area of $25 \%$ for two 30 -min consecutive images (Morel and Senesi 2002) and an eccentricity value close to 0.5 [average value of this parameter; Vila (2005)], a minimum value of 150 pixels
TABLE 1. Radiative parameters estimated by the ForTraCC algorithm.

Basic data

\begin{tabular}{lc}
\hline Date and time (UTC) & \\
Size of MCS & \\
Temperature threshold of \\
MCS definition $(235 \mathrm{~K})$ \\
$\quad$ Location data $(*)$ & Radiative parameters $(\mathrm{K})$ \\
\hline $\begin{array}{c}\text { Location of geometric MCS } \\
\text { center of mass }\end{array}$ & MCS mean brightness \\
Extreme location of MCS & temperature \\
MCS minimum brightness \\
temperature \\
location
\end{tabular}

* Lat and lon.

is obtained (considering the image resolution to be $4 \mathrm{~km} \times 4 \mathrm{~km}$, the minimum area will be $2400 \mathrm{~km}^{2}$ ) as a minimum size threshold for tracking a given MCS. In previous works, like Morel and Senesi (2002), a size threshold of $1000 \mathrm{~km}^{2}$ for Meteosat (around 70 pixels for GOES resolution) is established to track MCSs; so our selection appears to be a conservative assumption for describing the life cycle of a given MCS.

\section{b. The tracking technique}

By defining contiguous pixels that fulfill the minimum size and temperature thresholds defined in the previous section, regions that fulfill the brightness temperature threshold (MCS identification) and the minimum size requirements in a given image are identified.

Each of these cloud clusters (or pixel clusters) is identified with a number that will allow the generation of statistical information about each MCS (or cloud cluster) and its identification during the life cycle.

Some radiative parameters (based on the cloud-top temperature) are performed for each MCS detected in the previous process. These parameters (Table 1) are described extensively in Machado et al. (1998) and Vila and Machado (2004).

The tracking methodology is based on the tracking algorithm presented in Mathon and Laurent (2001). Tracking of convective clouds is based on an area overlap method. This technique simply assumes that a cloud at a later time corresponds to that at an earlier time when, considering the previous constraints of size and temperature, there are common pixels in consecutive images.

The comparison of successive satellite images is carried out "forward" and "backward" in time (Williams and Houze 1987; Mathon and Laurent 2001), so there 


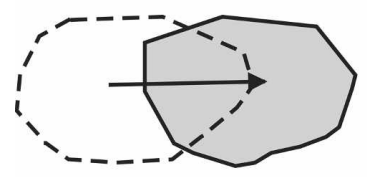

(a)

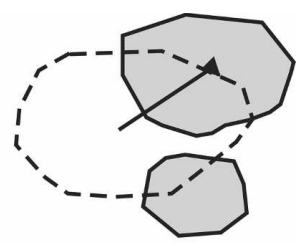

(b)

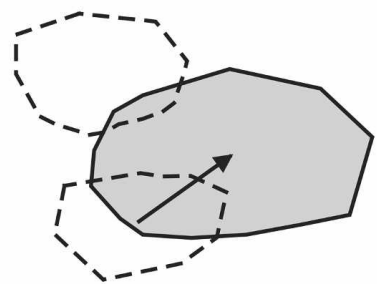

(c)

FIG. 2. Schematic representation of the tracking situations. White dotted figures represent MCSs in the first time step while gray figures represent the second time step. Arrows represent MCS evolution. Gray lines represent the previous time step evolution, and solid lines represent the actual evolution for (a) continuity, (b) splitting, and (c) mergers.

are five types of possible situations that can be obtained using this algorithm.

\section{1) Spontaneous generation (N)}

In a comparison of two successive frames, there is no overlap between the first frame (no MCS present) and the second one (an MCS is present); this situation is considered to be a spontaneous generation and the beginning of a new MCS life cycle.

\section{2) NATURAl Dissipation (NOR)}

Similar to the previous case, there is no overlap between the first frame (MCS is present) and the second one (MCS is not present); this situation is considered to be a natural dissipation of an MCS life cycle.

\section{3) Continuity (C)}

In a comparison of two successive frames (both forward and backward), there is an overlap of only one pair of MCSs. (Fig. 2a).

\section{4) SPLit (S)}

An example of this case is shown in Fig. 2b. This situation occurs when a forward comparison in time is performed. The first MCS (first time step) overlaps with more than one MCS in the second time step. In this case, a split occurs and the larger overlapping surface between the first MCS and the MCS found in the second step determines which MCS is chosen to con- tinue the MCS life cycle, while the other one represents the initiation of a new MCS cycle (initiation by split).

\section{5) Merger (M)}

Similar to the previous case, except here a backward comparison is performed. There is a match between more than one MCS in the first time step and only one in the second frame. In this case, a merging situation is present (Fig. 2c). The larger overlapping surface determines which MCS is chosen to continue the MCS life cycle, while the others (in case there is more than one MCS present) represent a dissipation of the life cycles of those MCSs (dissipation by merging).

\section{c. The forecast technique}

The methodology used to forecast the MCS trajectory (displacement of the geometrical center of mass) and the life cycle phase and area (growth-decay) of a given MCS is presented in this section.

\section{1) MCS CENTER OF MASS DISPLACEMENT ESTIMATION}

Starting from the identification and tracking of a given MCS in three consecutive times- $t-2 \Delta t, t-\Delta t$, and $t$-along a life cycle, the estimated MCS displacement is performed by applying the following procedure. The estimated displacement vector in the previous time interval, $\mathbf{V}(t-1)$, is performed considering the displacement of the geometrical center of mass between $t-2 \Delta t$ and $t-\Delta \mathrm{t}$. Considering that this displacement does not change very much (in magnitude or direction) at such a time scale (30 min), a predicted velocity $\mathbf{V P}(t)$ is generated. Simultaneously, the real MCS displacement, $\mathbf{V}(t)$, is also calculated considering the $t-\Delta t$ and $t$ time intervals. The estimation of the MCS vector displacement $\mathbf{V E}(t+1)$ is performed as $\mathbf{V}(t)$ plus the difference between the real displacement and the predicted velocity $[\Delta \mathbf{V}(t)=\mathbf{V}(t)-\mathbf{V P}(t)]$ in the last time step. This procedure, which conserves the MCS's shape and size, is presented in Fig. 3.

If two successive time steps for the same MCS are not available, the MCS vector displacement is estimated as the displacement of the center of mass between $t-\Delta t$ and $t$.

This procedure is applicable only if the continuity condition (C) is fulfilled, because splitting and merging of an MCS introduces a nonrealistic MCS center of mass displacement. In these cases, as well as in the case of spontaneous generation $(\mathrm{N})$, the displacement is performed as a distance-weighted average displacement of the neighboring MCS. 


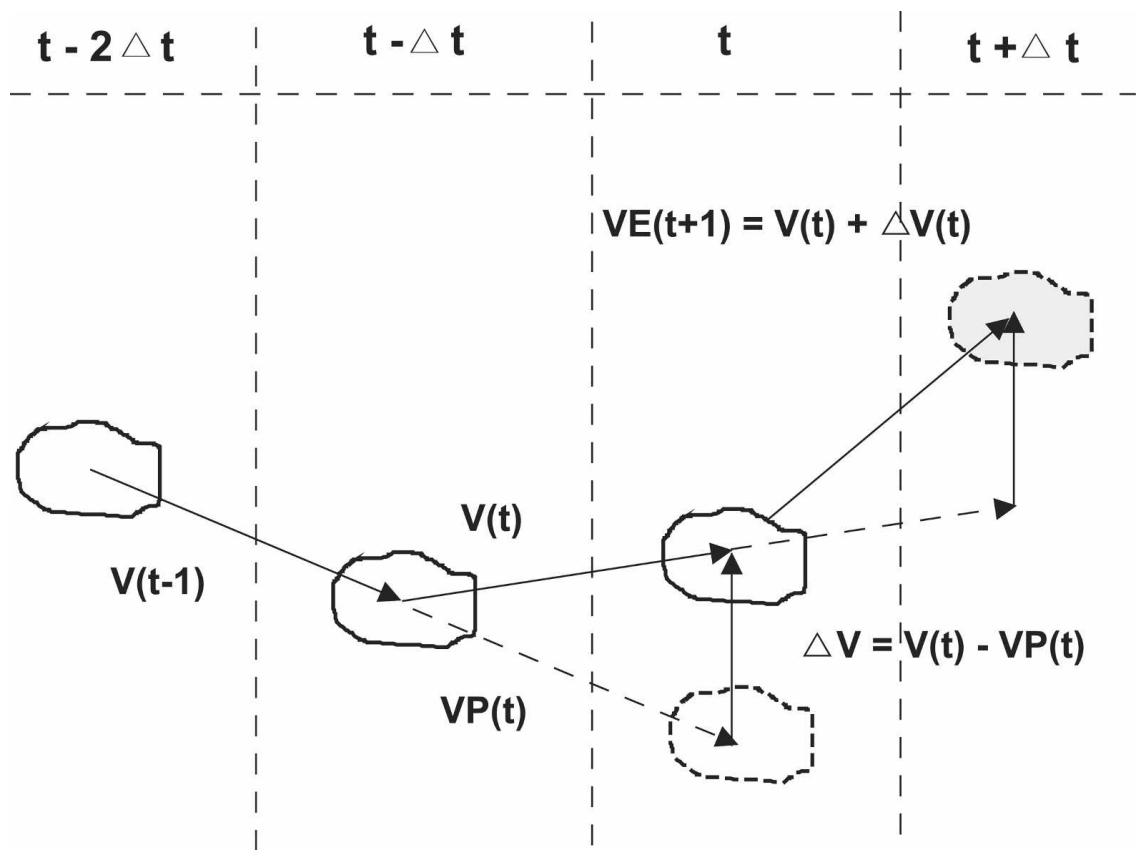

FIG. 3. Schematic representation of the displacement forecast for the MCS's center of mass. The vertical dotted lines separate the different time steps. The dashed MCS indicates the forecasted position of the cloud system.

\section{2) MCS LIFE CYCLE PHASE (GROWTH-DECAY)}

The life cycle phase of a given MCS (growth-decay) is evaluated through the normalized area expansion. This parameter is expressed as $\Delta E=1 / A(\delta A / \delta t)$ (see Machado et al. 1998; Machado and Laurent 2004), where $A$ is the area of a given MCS at a given time $\left(T_{\mathrm{ir}}<235 \mathrm{~K}\right)$. Positive values indicate an expansion process (growth) and negative values indicate that the is MCS decreasing in size.

To determine the life cycle phase of a given MCS (growth or decay), a statistical study of the $\Delta E$ parameter during the life cycle of all MCSs generated during the Wet Season Atmospheric Mesoscale Campaign (WETAMC) of the Large-Scale BiosphereAtmosphere Experiment (LBA) (Silva Dias et al. 2002) was performed.

Only spontaneously generated life cycles with no merging or splitting during the life cycle and with normal dissipation were used to obtain mean $\Delta E$ values for different life time cycles.

The objective of this section is to determine the statistical parameters of a general model that will represent the MCS life cycle to be used in the forecast process. The basic idea is expressed in Machado and Laurent (2004). An MCS life cycle can be estimated using the following equation:

$$
A(t)=\alpha e^{a t^{2}+b t+c},
$$

where $A(t)$ is the MCS area at the time $t$ of the life cycle and $a, b$, and $c$ are parameters to be defined according to the life cycle's duration.

Based on Eq. (1), $\Delta E$ are typically straight lines:

$$
\Delta E=1 / A(\partial A / \partial t)=a t+b
$$

where $a$ (slope) and $b$ (interception) depend on the MCS's total life cycle duration. A statistical study focusing on the assessment of these two coefficients according to the life cycle duration of a typical MCS was conducted.

Based on the MCS statistics obtained from the WETAMAC experiment, four life cycle duration classes were used: families with life cycles with a duration of less than $2 \mathrm{~h}$, those lasting between 2 and $4.4 \mathrm{~h}$, between 4.4 and $8.4 \mathrm{~h}$, and those lasting longer than $8.4 \mathrm{~h}$. The selection of nonuniform categories is related to the fact that, for statistical purposes, the numbers of cases of each category should be similar. In this study [and other related results for other regions of the world; i.e., Mathon and Laurent (2001); Morel and Senesi (2002)], short-lived MCSs are more frequent than long-lived MCSs, while most long-lived MCS have larger probabilities of having splits and mergers during their life cycles and are consequently excluded from this statistical analysis. 


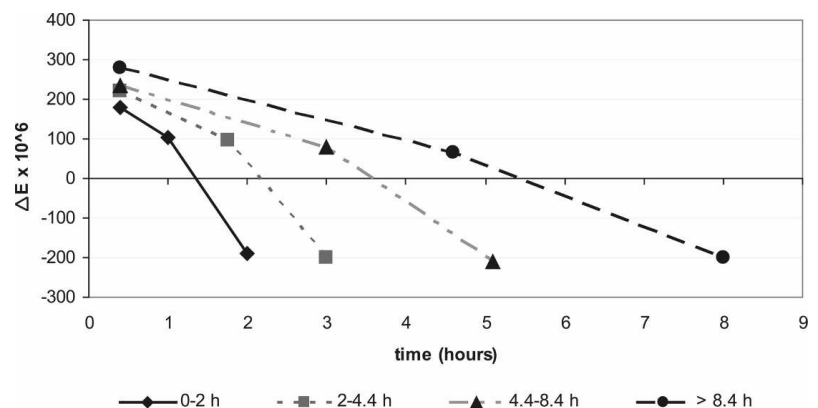

FIG. 4. Mean value of $\Delta E\left(\times 10^{6} \mathrm{~s}^{-1}\right)$ at initiation, maturation, and dissipation of an MCS for different life durations: 0-2, 2-2.4, 4.4-8.4, and more than $8.4 \mathrm{~h}$.

For each one of these four groups, three mean values of $\Delta E$ was evaluated: (a) the mean value of $\Delta E$ in the first instant of life of each MCS (initiation), (b) the mean value of $\Delta E$ in the middle of the MCS life time (maturation), and (c) the mean value of $\Delta E$ in the instant of the MCS end (dissipation). These points are plotted in Fig. 4 and connected with lines. These points can be fitted with a linear equation $(r>0.90$ in all cases), so slope and intercept can be obtained through a linear regression analysis for each life cycle duration class.

In the general case, as each class can be fitted through a linear equation, it is possible to obtain, using an interpolation process, a family of curves in $T-\Delta E$ space that represents the evolution of all possible MCSs (from initiation to dissipation). Using this model, each point in that space $\left(T_{i}, \Delta E_{i}\right)$ is related with an unique curve that represents the life cycle evolution of a particular MCS at a particular moment during a lifetime $\left(T_{i}=\right.$ time elapsed from initiation to current time).

This MCS life cycle model (family of curves) is used to determine the probable evolution of each MCS in the following way. Consider that an MCS is being tracked along its life cycle and the last available information about its size is known at time $t_{1}$. The elapsed time from the spontaneous generation $\left(t_{0}\right)$ to the last available image is $T_{1}=t_{1}-t_{0}$. At the same time, $\Delta E_{1}$ can be computed for the last two consecutive images. Using the above-mentioned approach, a unique linear equation is selected from the family of curves that represents the possible evolution of all MCSs. The slope and interception ( $a_{\text {est }}$ and $\left.b_{\text {est }}\right)$ represent the life cycle evolution of that particular MCS and it is used for the size forecast. This is obtained by applying these coefficients to the next time step in Eq. (2) $\left(\Delta E=a_{\text {est }} t_{1+1}+b_{\text {est }}\right)$. If $t_{1}=30 \mathrm{~min}$, the initial expansion is used to estimate the size evolution for that MCS as suggested in Machado and Laurent (2004). In this process, the size of the MCS can be modified but the

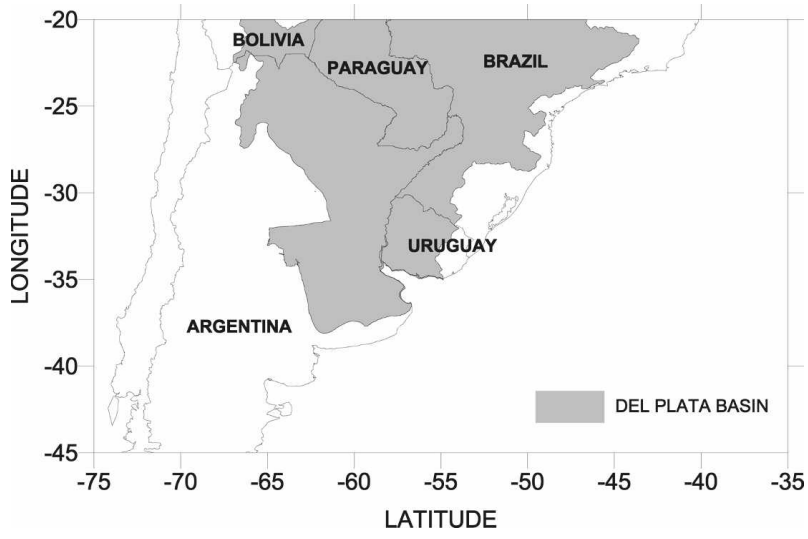

FIG. 5. The Del Plata basin region.

shape remains unchanged (only affected by scale factors).

A similar statistical analysis is applied to estimate the MCS's brightness temperature evolution. We have hypothesized that the size evolution is linearly followed by a cloud-top variation $\left(\Delta T=\delta T_{\min } / \delta t\right)$. A linear relationship is performed between $\Delta E$ and $\Delta T$, which is also applied to the forecast procedure in order to obtain the evolution of the minimum temperature.

This forecast technique can be performed only if continuity is observed for a given MCS. Spurious values of the $\Delta E$ result from splitting or merging are not considered, and, in this case, the previous time step values $\left(a_{\text {est }}\right.$ and $\left.b_{\text {est }}\right)$ are used for the forecast process.

\section{Validation of the nowcasting methodology}

The forecast process is carried out by considering three parameters: the MCS displacement estimation, as a function of the displacement evolution at the previous time; the MCS area evolution based on the linear regression shown in Fig. 4; and the MCS minimum brightness temperature temporal rate.

The validation process is performed using two methodologies: the first one is based on the MCS size evolution and the position of each MCS. This technique is applied only in cases of continuity (no merge, no split). The second one is based on contingency tables and statistical parameters for the whole image. Because many life cycles have mergers and splits, this validation procedure takes into account all of the cases.

\section{a. Validation data and methodology}

This nowcasting tool was applied to evaluate the displacement and MCS life cycle over the Del Plata basin in South America (Fig. 5) for up to 120 min with 30-min time intervals during December 2002-January 2003. 


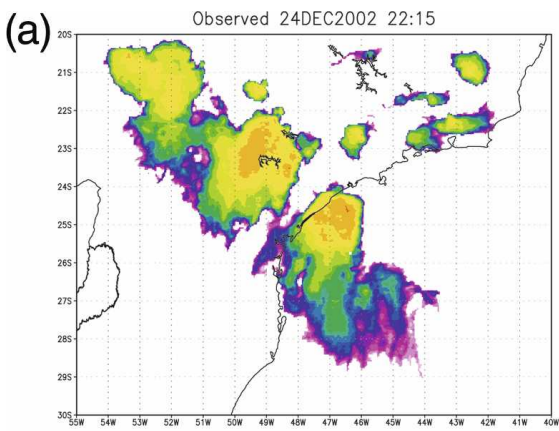

(c)

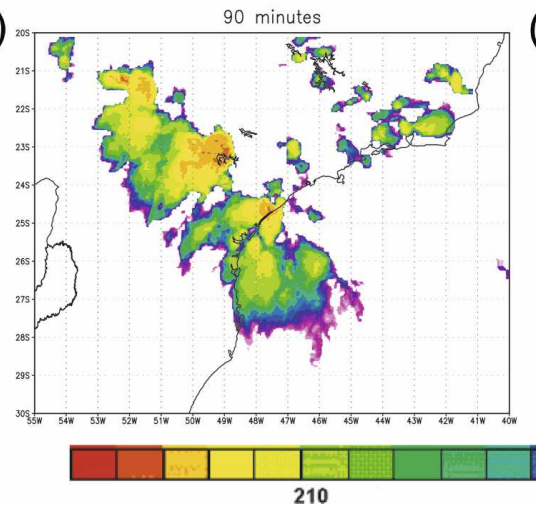

(b)

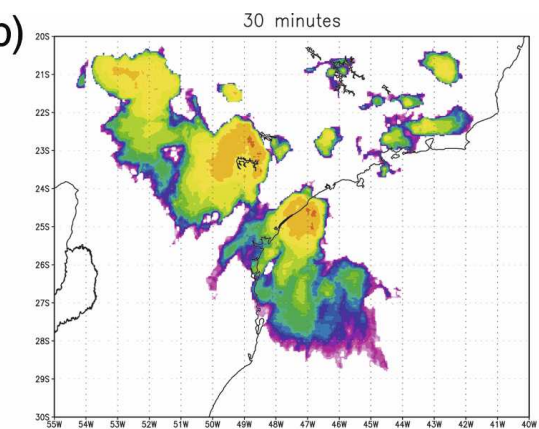

(d) 120 minutes

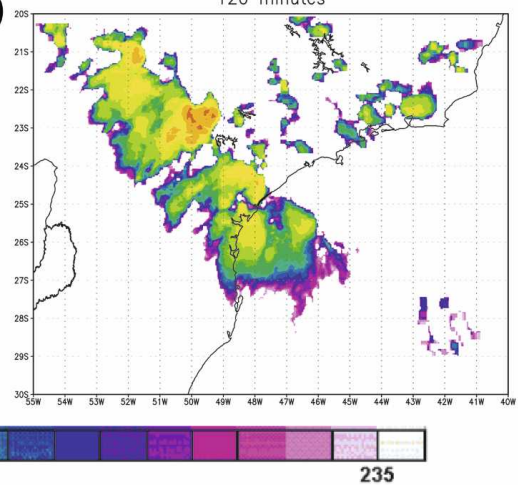

FIG. 6. Cold cloud cover at 2245 UTC 24 Dec 2002 for a subset of the Del Plata basin in southeastern Brazil. (a) The observed image, (b) the 30-min forecast image, (c) the 90-min forecast image, and (d) the 120-min forecast image.

Figure 6 shows an example of the FORTRACC output. An image subset covering the northeastern region of the Del Plata basin at 2215 UTC 24 December 2002 (Fig. 6a) is presented. A color table was used to show pixels with brightness temperatures below $235 \mathrm{~K}$. Small MCSs (below 150 pixels) and pixels warmer than $235 \mathrm{~K}$, which are not considered in the ForTraCC procedures, were excluded to facilitate the visualization of the region of interest. The other three panels show the results of the forecast at the same time, for different time leads (30, 90, and $120 \mathrm{~min})$. For 30-min forecast lead time (Fig. 6, top right), the predicted image at 2215 UTC 24 December 2002 was computed from the initial image observed at 2145 UTC of the same day, and so on for the other forecast lead times.

The presence of a large MCS with three very well defined cold tops and a smaller group of convective systems located to the northeast of the large MCS (Fig. 6 ) is well represented by the predicted images. It is clear that the forecasting technique can resolve the main characteristics of the observed image (such as position and size), especially until $90 \mathrm{~min}$. In the last image whose forecast range is $120 \mathrm{~min}$, larger disparities are observed among the position, size, and intensity between the observed and predicted MCSs although the main cloud cluster has a similar structure.

\section{b. MCS size and temperature evolution}

This verification process is carried out by comparing the evolution of the main forecast parameters for each MCS with the observed ones. Only MCSs with no splits-mergers during the forecast time were taken into account in this verification process.

Figure 7 shows the size and minimum temperature evolution for a single MCS present at 0345 UTC 6 January 2005. Solid lines represent the observed values for both parameters while dotted lines represent the fore-

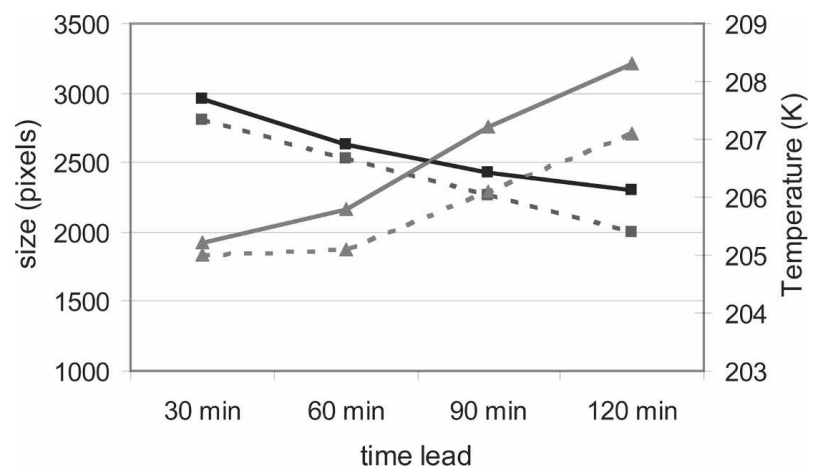

FIG. 7. Size (black lines) and minimum temperature (gray lines) evolutions for a single MCS at 0345 UTC 6 Jan 2005. Solid lines represent the observed values for both variables while dotted lines represent the forecast obtained with ForTraCC. 

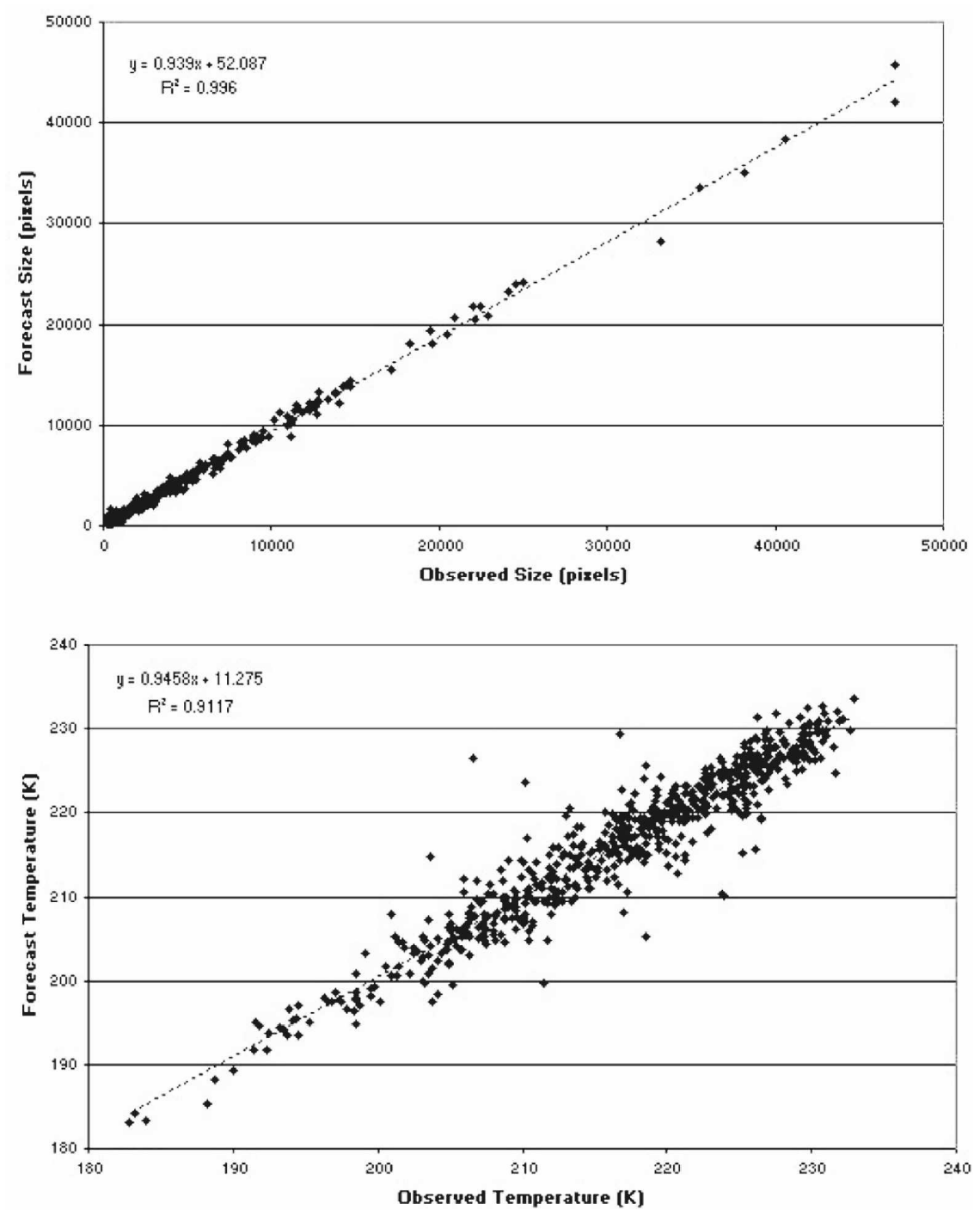

FIG. 8. (top) The observed and forecasted MCS sizes during the validation period for a 30-min forecast. (bottom) As in (top) but for the minimum temperature.

cast obtained with ForTraCC for different time leads. In this case, the selected MCS (located over the Atlantic Ocean) is entering the dissipation phase (the MCS size is decreasing and the minimum brightness temperature is increasing; solid lines in Fig. 7) and the forecast tends to underestimate this process.

The comparison between the observed and forecasted parameters, shown in the previous example for a single MCS, was applied to all MCSs for the period between 5 and 11 January 2003.

Figure 8 shows the scatter diagram of the observed and forecasted size and minimum brightness temperature for 30-min forecasts in both cases; good agreement between the observed and forecasted values is observed. The minimum temperature shows a larger dis- persion for the size, but both parameters exhibit a large correlation coefficient $(r>0.9)$ and the slope of the first-order regression is close to 1 (observation $=$ forecast). The size and eccentricity evolutions (not shown) exhibit a similar behavior. Obviously, a decrease in the forecast skill is observed for the 120-min forecasts. The model tends to dissipate the MCS too quickly (and warmer minimum temperatures are achieved). This process can be observed in Fig. 9. In this case, the slope of the first-order regression is lower than 1 and the correlation coefficient is lower than in the previous case (larger dispersion). The reduction in the number of cases for the 120-min forecast range is due to the continuity restriction applied and the smaller numbers of MCSs having life cycles up to $120 \mathrm{~min}$. 

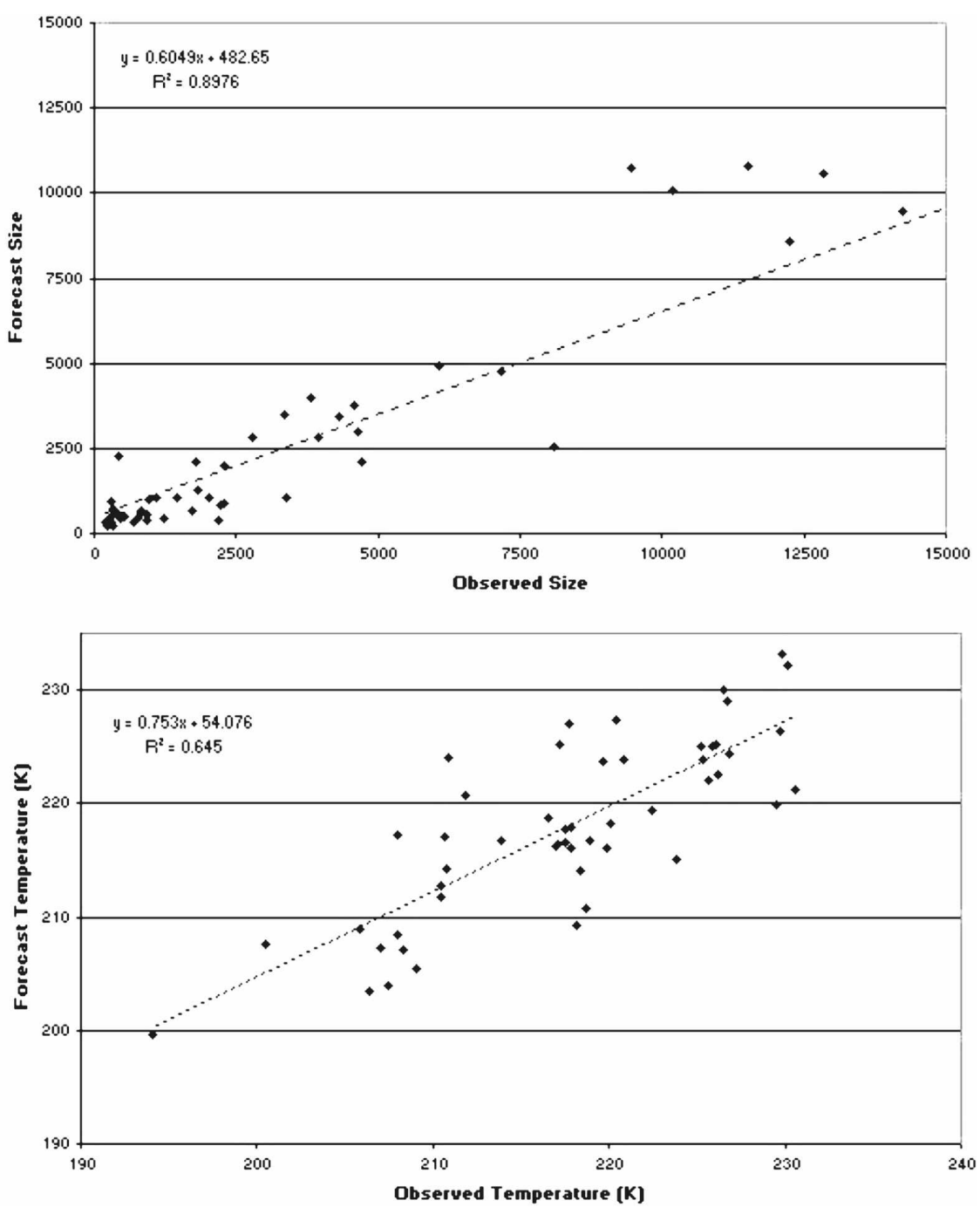

FIG. 9. Same as in Fig. 8 but for 120-min forecast.

The size and minimum temperature forecast is also compared with a conservative assumption: where the MCS maintain its size and position unchanged throughout the time. This case will be called, hereafter, the nonforecast. Since there are no other objective or subjective methodologies over South America to compare with, this test was applied to verify if this assumption is better (or not) than the ForTrACC technique mainly when the lag time increases and the forecast quality decreases. Table 2 illustrates this situation: in all lead times the size mean bias ( $\Delta$ Size, expressed in \%) and the minimum temperature bias $\left(\Delta T_{\min }\right)$ exhibit better results for the forecast model than for the nonforecast situation. It also can be show, as in the previous analysis, that there is a lack of quality for longer lead times.

Another validation test was performed for analyzing the displacement of the MCS center of mass. This test was carried out by taking into account the distance $(\mathrm{km})$ between the observed and forecast centers of mass. These results are shown in Fig. 10. The histograms describe the relative frequencies of the distance classes. In the first case (30-min forecast), the mean distance between the observed and forecasted mass center is around $21 \mathrm{~km}$ (around 5 pixels), while in the second case (120 $\mathrm{min}$ ) this value reaches $81 \mathrm{~km}$ (around 20 pixels). Note that the forecast methodology does not take into account the deformation of the MCS during its life cycle.

\section{c. Contingency tables and categorical statistics}

Because MCS life cycles with frequent splits and mergers are very hard to validate, a complementary validation methodology based on contingency tables was also performed to measure the ForTraCC skill. In 
TABLE 2. Mean bias $(\Delta)$ of the size (expressed in \% to show the relative variations in size) and minimum temperature for the forecast and nonforecast situations. Each row represents different lead times (min).

\begin{tabular}{cccccccc}
\hline \hline & \multicolumn{3}{c}{ Forecast } & & \multicolumn{3}{c}{ Nonforecast } \\
\cline { 2 - 4 } \cline { 6 - 8 } Time (min) & $\Delta$ size $(\%)$ & $\Delta T_{\text {med }}(\mathrm{K})$ & $\Delta T_{\min }(\mathrm{K})$ & & $\Delta$ size $(\%)$ & $\Delta T_{\text {med }}(\mathrm{K})$ & $\Delta T_{\min }(\mathrm{K})$ \\
\hline 30 & $-1.87 \%$ & 0.25 & 0.35 & & $-3.17 \%$ & 0.34 & 0.46 \\
60 & $-4.20 \%$ & 0.59 & 0.66 & & $-8.24 \%$ & 0.50 & 0.62 \\
90 & $-7.80 \%$ & 0.75 & 0.52 & & $-23.50 \%$ & 0.79 & 0.93 \\
120 & $1.81 \%$ & 0.75 & -0.35 & & $-28.80 \%$ & 1.00 & 0.88 \\
\hline
\end{tabular}

this case, the contingency table is performed for the whole image and it is not possible to know how the forecast works for a particular MCS. This methodology shows the frequency of "yes" and "no" forecasts and occurrences (pixels below $235 \mathrm{~K}$, in this case). The four combinations of forecasts (yes or no) and observations (yes or no) are defined as follows:

(a) Hit: The brightness temperature of the forecasted and observed pixel is below $235 \mathrm{~K}$.

(b) Correct negative: The brightness temperature of the forecasted and observed pixel is above $235 \mathrm{~K}$.

(c) Miss: The brightness temperature of the forecasted pixel is above $235 \mathrm{~K}$ but the observed temperature of that pixel is below $235 \mathrm{~K}$.
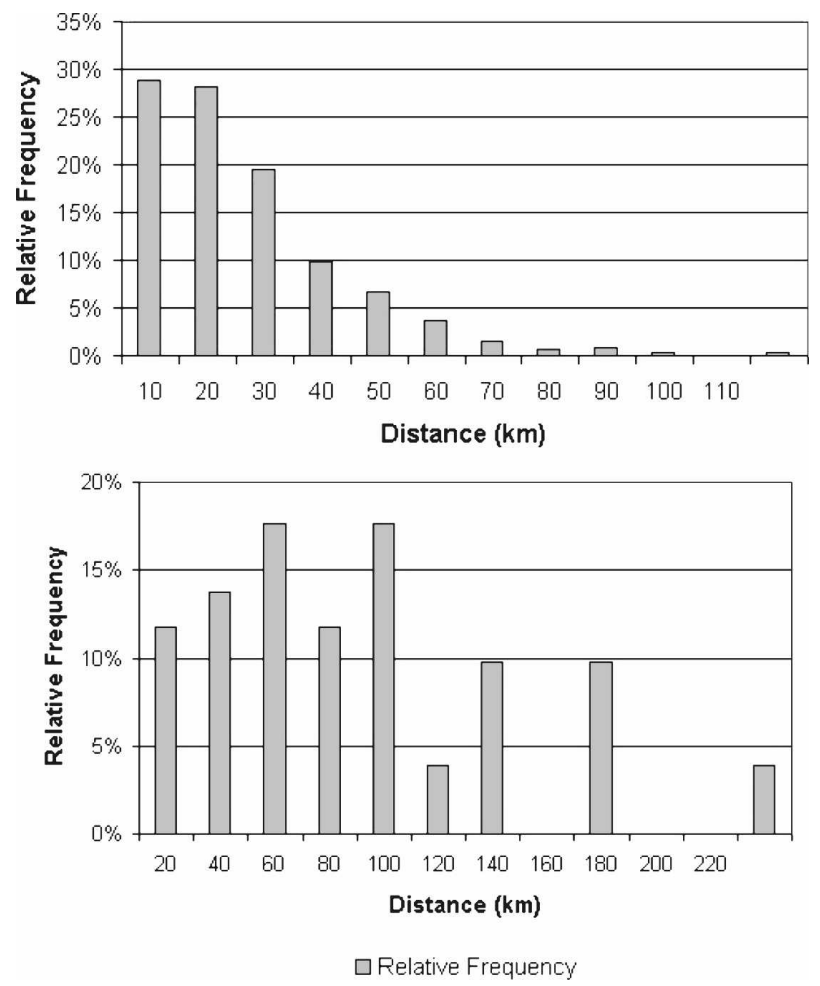

FIG. 10. Relative frequency of distance classes between observed and forecasted mass centers: (top) 30- and (bottom) 120min forecasts. (d) False alarm: The brightness temperature of the forecasted pixel is below $235 \mathrm{~K}$ but the observed temperature is above $235 \mathrm{~K}$.

A large variety of statistical indexes were computed from the contingency table to describe particular aspects of forecast performance like accuracy (ACU), bias score (BIAS), probability of detection (POD), and false alarms rate (FAR). For the definitions of these terms, please refer to, for example, Wilks (1995).

ACU is the fraction of the correctly forecasted pixels compared with the total number of pixels in a given image. The more accurate forecast corresponds to ACU $=1$ and this parameter can be misleading since it is heavily influenced by the most common category (correct negative).

The BIAS score measures the error between the number of pixels predicted as MCS $\left(T_{\mathrm{ir}}<235 \mathrm{~K}\right)$ and the number of pixels observed as MCS $\left(T_{\mathrm{ir}}<235 \mathrm{~K}\right)$. In this case no bias corresponds to a value equal to 1 . This index determines if the forecast methodology tends to overestimate (BIAS $>1$ ) or underestimate (BIAS $<1$ ) the number of pixels with $T_{\mathrm{ir}}<235 \mathrm{~K}$. Nevertheless, it does not determine how well the pixels are adjusted for in space, since this index only computes a relative frequency.

To avoid the inclusion of correct negative reinforcements, POD and FAR were included in the study. The POD allows for us knowing the fraction of the observed pixels that has been correctly predicted. This value is not sensitive to the number of false alarms (a pixel with $T_{\mathrm{ir}}<235 \mathrm{~K}$ is predicted but it is not observed), so it is used in conjunction with the FAR. In this case, this index indicates the ratio of false alarms with regard to the number of well-predicted pixels $\left(T_{\mathrm{ir}}<235 \mathrm{~K}\right)$.

Figures 11a and 11c show an example of the time evolution of the number of MCS pixels observed and predicted $\left(T_{\mathrm{ir}}<235 \mathrm{~K}\right)$ for the entire image for 30- and 120-min forecast lead times for the period 6-11 January 2003. It can be observed that, in both cases, the diurnal cycle is correctly predicted. The mean amount of observed pixels with $T_{\mathrm{ir}}<235 \mathrm{~K}$ is around $5 \%$ higher than the mean value of the predicted ones for the same pe- 

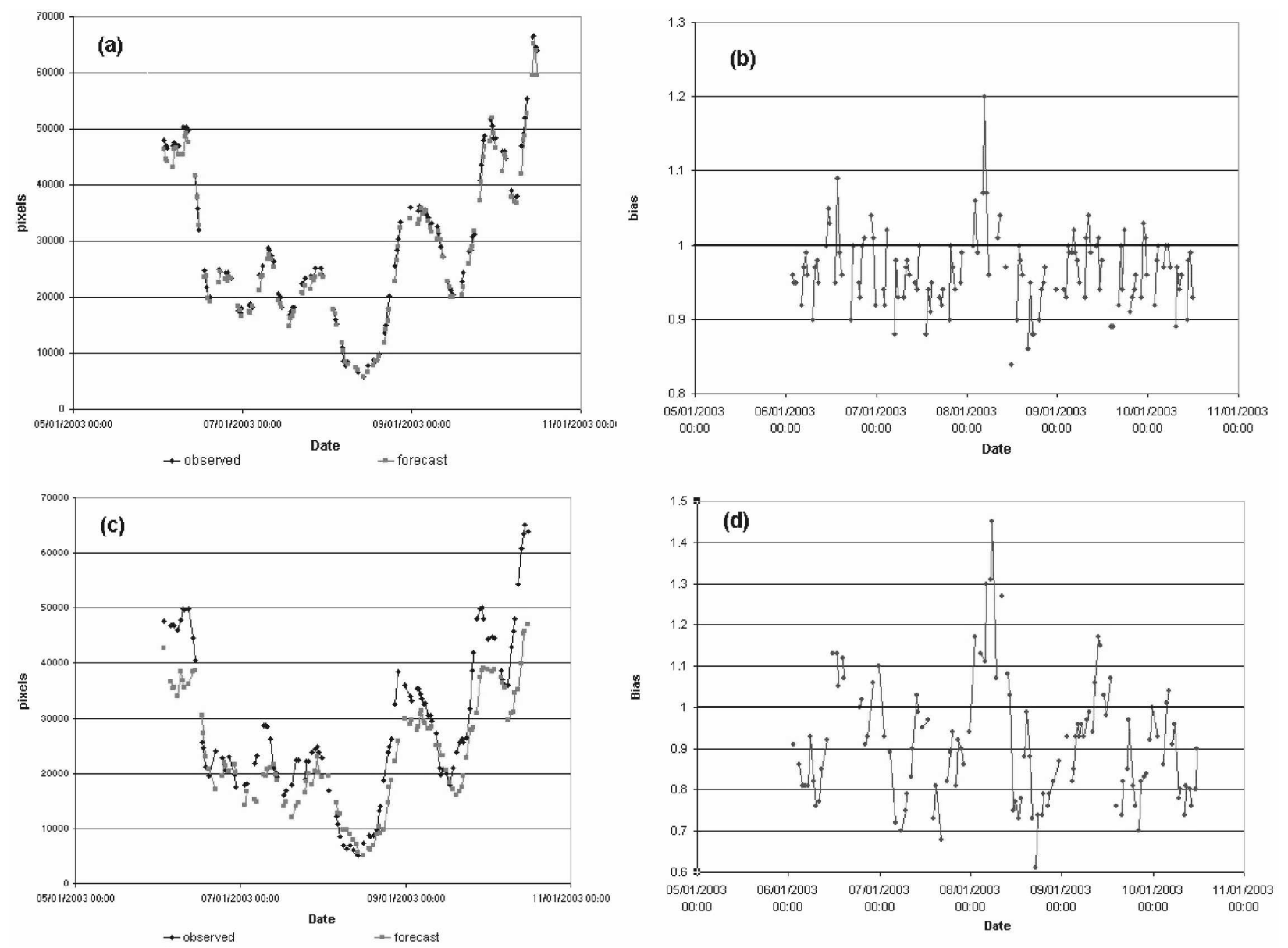

FIG. 11. (a) Number of observed and forecasted MCS pixels per image during the period 6-11 Jan 2003 for the 30-min forecast range. (b) BIAS score per image for the same period for the 30-min forecast range. (c) As in (a) but for the 120-min forecast range. (d) As in (b) but for the 120-min forecast range. Blanks in the solid line correspond to missing data (the GOES-8 southern scan was not available).

riod. For the period between 25 and 30 December 2002 (not shown), the behavior is similar. The large-scale environment and the convective activity are very important factors in determining these values. Nevertheless, in both cases, a small underestimate in the number of predicted pixels (on average) is observed. This fact can be observed in the temporal series of BIAS (Figs. $11 \mathrm{~b}$ and $11 \mathrm{~d}$ ), where values below 1 are more frequent that larger ones. A less favorable situation is obtained for 120-min forecast lead time (Figs. 11c and 11d), where the larger underestimations are present. This result suggests that the proposed model tends to dissipate MCSs more quickly than observed systems. This result can be explained by the fact that the proposed model of MCS evolution predicts only an initiation, a maturation, and a dissipation phase. In the real world, the behavior of MCSs is more complex with frequent regenerations, splits, mergers, and spontane- ous generation of new convective systems that are not considered in the proposed model. Nevertheless, the forecast reflects the same diurnal cycle structure of the convection and a quite good performance for up $90 \mathrm{~min}$.

Table 3 shows the statistical indexes for 30, 60, 90, and $120 \mathrm{~min}$. The accuracy index (ACU) shows larger values for the 30-min forecast lead time. The mean value surpasses 0.98 . In other words, $98 \%$ of the pixels have been correctly predicted. However, this value is high because most of the values correspond to correct negative pixels $\left(T_{\mathrm{ir}}>235 \mathrm{~K}\right)$. The POD, for a $30-\mathrm{min}$ forecast, reaches approximately 0.77 and the FAR is around 0.20 . In other words, while $77 \%$ of the observed pixels corresponding to $T_{\mathrm{ir}}<235 \mathrm{~K}$ were correctly predicted, $20 \%$ of the predicted pixels were not observed. On the right side of Table 2 (120-min forecast), the ACU index is about 0.96 for both periods of analysis 
TABLE 3. ACU, BIAS, POD, and FAR for 30-, 60-, 90-, and 120-min forecast lead times for the period 6-11 Jan 2003.

\begin{tabular}{lcccc}
\hline \hline & $30 \mathrm{~min}$ & $60 \mathrm{~min}$ & $90 \mathrm{~min}$ & $120 \mathrm{~min}$ \\
\hline ACU & 0.98 & 0.98 & 0.97 & 0.96 \\
BIAS & 0.96 & 0.95 & 0.91 & 0.87 \\
POD & 0.77 & 0.64 & 0.54 & 0.44 \\
FAR & 0.20 & 0.32 & 0.41 & 0.49 \\
\hline
\end{tabular}

(December and January) and although around $96 \%$ of the pixels have been correctly predicted (also considering the correct negative), POD and the FAR appear to be slightly too far from the ideal case.

This analysis complements the previous one showing similar results independently of the applied methodology.

\section{Summary and conclusions}

The ForTraCC technique for detection, tracking, and short-term forecasting of MCSs using satellite images has been presented. This technique, which is completely automated, is composed of four independent modules: (a) cloud detection defined by a size and temperature threshold, (b) observation of MCS physical characteristics based on cold cloud tops, (c) a tracking algorithm based on overlapping images, and (d) MCS evolution forecasts.

The physical basis of the forecasting algorithm is based on the fact that the MCS area's evolution (and its minimum temperature) follows a typical pattern of behavior, which only depends on the total life cycle of that MCS. The propagation is based in the MCS's movement in the last $30 \mathrm{~min}$, corrected by former errors in previous time steps.

The size evolution model is based on the work in Machado and Laurent (2004) using MCS life cycle statistics from the Wet-Season Atmospheric Mesoscale Campaign (WETAMC) of the Large-Scale BiosphereAtmosphere Experiment (LBA).

A statistical verification was carried out by comparing real images and forecasted images. The main results are summarized by the following points:

(a) Considering the forecasted and observed pixels with brightness temperatures below $235 \mathrm{~K}$ (independently of their positions), it is observed that the model tends to underestimate the number of pixels; this underestimation, on average, increase with longer forecast lead times.

(b) The proposed methodology follows the diurnal cycle of cold cloud cover $\left(T_{\text {ir }}<235 \mathrm{~K}\right)$ cloud coverage with acceptable accuracy in amplitude and phase. (c) The mean accuracy (ACU) obtained with this technique is about $95 \%$. This high value is mainly due to the correct prediction of no MCS occurrence. In the case of POD and FAR (which do not include this case), a gradual lost of quality is observed. POD decreases and FAR increases with forecast range.

(d) The behavior of individual MCSs shows good agreement between the observations and forecasts of size and minimum temperature for a 30-min forecast range, with a lose of quality for longer forecast range.

The information generated by ForTraCC is very useful for different stakeholders, such as energy distributors and emergency management agencies, among others. Updated information about MCS evolution over South America can be obtained online (http:// satelite.cptec.inpe.br). This service has been working operationally since 2004.

This methodology is a first approach to this problem, since the families selected for the statistical study have some bias (no splitting and/or no merging during the entire life cycle). Nevertheless, the results obtained in this study are quite reasonable and they encourage us to continue with this approach in trying to find regionalized relationships to improve our nowcasting and also for studying the cases of more complex MCS behavior (regenerations, external forcing, etc.) In the future, this methodology should be combined with a mesoscale numerical model to include new MCS developments and to take into account the merging, splitting, and regeneration processes in the evolution of MCS life cycles.

Acknowledgments. Satellite imagery was obtained from Divisão de Satélites e Sistemas Ambientais/ Centro de Previsão de Tempo e Estudos Climáticos (DSA/CPTEC). The first author acknowledges Eletropaulo and Elektro for the financial support. In memory of Henri Laurent (1958-2007), a great researcher and best friend.

\section{REFERENCES}

Carvalho, L., and C. Jones, 2001: A satellite method to identify structural properties of mesoscale convective systems based on the maximum spatial correlation tracking technique (MASCOTTE). J. Appl. Meteor., 40, 1683-1701.

Corfidi, S. F., J. H. Meritt, and J. M. Fritsch, 1996: Predicting the movement of mesoscale convective complexes. Wea. Forecasting, 11, 41-46.

Houze, R. A., 1977: Structure and dynamics of a tropical squallline system. Mon. Wea. Rev., 105, 1540-1567.

Laurent, H., L. A. T. Machado, C. Morales, and L. Durieux, 2002: Characteristics of Amazonian mesoscale convective systems observed from satellite and radar during the WETAMC/ 
LBA experiment. J. Geophys. Res., 107, 8054, doi:10.1029/ 2001JD000337.

Le Barbé, L., T. Label, and D. Tapsoba, 2002: Rainfall variability in West Africa during the years 1950-90. J. Climate, 15, 187202.

Machado, L. A. T., and H. Laurent, 2004: The convective system area expansion over Amazonia and its relationships with convective system life duration and high-level wind divergence. Mon. Wea. Rev., 132, 714-725.

_ J.-Ph. Duvel, and M. Desbois, 1993: Diurnal variations and modulation by easterly waves of the size distribution of convective cloud clusters over West Africa and Atlantic Ocean. Mon. Wea. Rev., 121, 37-49.

— W. B. Rossow, R. L. Guedes, and A. W. Walker, 1998: Life cycle variations of mesoscale convective systems over the Americas. Mon. Wea. Rev., 126, 1630-1654.

Maddox, R. A., 1980: Mesoscale convective complex. Bull. Amer. Meteor. Soc., 61, 1374-1387.

Mapes, B. E., and R. A. Houze Jr., 1992: An integrated view of 1987 Australian monsoon and its mesoscale convective systems. Part I: Horizontal structure. Quart. J. Roy. Meteor. Soc., 118, 927-963.

Mathon, V., and H. Laurent, 2001: Life cycle of the Sahelian mesoscale convective cloud systems. Quart. J. Roy. Meteor. Soc., 127, 377-406.

Morel, C., and S. Senesi, 2002: A climatology of mesoscale convective systems over Europe using satellite infrared imagery. I: Methodology. Quart. J. Roy. Meteor. Soc., 128, 1953-1971.

Scofield, R., R. Kuligowski, and C. Davenport, 2004: The use of the Hydro-Nowcaster for mesoscale convective systems and the Tropical Rainfall Nowcaster (TRaN) for landfalling tropical systems. Preprints, Symp. on Planning, Nowcasting, and Forecasting in the Urban Zone, Seattle, WA, Amer. Meteor. Soc., 1.4.
Silva Dias, M. A. F., and Coauthors, 2002: Cloud and rain processes in a biosphere-atmosphere interaction context in the Amazon region. J. Geophys. Res., 107, 8072, doi:10.1029/ 2001JD000335.

Torres, J. C., 2003: Heavy rainfall mesoscale convective systems in northern and middle Argentina (in Spanish). Ph.D. thesis, Facultad de Ciencias Exactas y Naturales, Universidad de Buenos Aires, Buenos Aires, Argentina, 213 pp.

Velasco, I., and G. Necco, 1980: Valores medios, extremos y desviaciones estandar de datos aerológicos de la república Argentina (Mean, extreme and standard deviations values of rawinsonde data over Argentina). Departamento de Meteorología, Facultad de Ciencias Exactas y Naturales, Universidad de Buenos Aires, Buenos Aires, Argentina.

_, and J. M. Fritsch, 1987: Mesoscale convective complexes in the Americas. J. Geophys. Res., 92, 9591-9613.

Vila, D. A., 2005: Sistemas convectivos precipitantes de mesoescala sobre Sudamerica: Ciclos de vida y circulación en gran escala asociada (Rainy mesoscale convective systems over South America: Life cycle and the associated large scale environment). Ph.D. thesis, Facultad de Ciencias Exactas y Naturales, Universidad de Buenos Aires, Buenos Aires, Argentina, $132 \mathrm{pp}$.

- and L. A. T. Machado, 2004: Shape and radiative properties of convective systems observed from infrared satellite images. Int. J. Remote Sens., 25, 4441-4456.

Wilks, D. S., 1995: Statistical Methods in the Atmospheric Sciences: An Introduction. Academic Press, 467 pp.

Williams, M., and R. A. Houze Jr., 1987: Satellite-observed characteristics of winter monsoon cloud clusters. Mon. Wea. Rev., 115, 505-519.

Woodley, W. L., C. G. Griffith, J. S. Griffin, and S. C. Stromatt, 1980: The inference of GATE convective rainfall from SMS-1 imagery. J. Appl. Meteor., 19, 388-408. 